\title{
SUBSMELL: Multimedia with a Simple Olfactory Display
}

\author{
Chomtip Pornpanomchai, Arinchaya Threekhunprapa, Krit Pongrasamiroj, \\ and Phichate Sukklay
}

\author{
Mahidol University, Department of Computer Science, Faculty of Science, \\ Rama 6 Road, Rajchatavee, Bangkok 10400, Thailand \\ \{cccpp, u4788241,u4788324, ccpsk\}@mahidol.ac.th
}

\begin{abstract}
The idea of adding the SubSmell logo to the movie for describing the scent of each event in the movie has been proposed to improve the current way of seeing movies, which can perceive only pictures and sound. Using the SubSmell, the audience can smell the movie. The audiences need a SubSmell application to read a SubSmell and an olfactory display in order to release scent.

There are two main parts in SubSmell system, which are an olfactory display and a SubSmell application. An olfactory display consists of a control box and four smell boxes with four fans. Fans will be turned on and release scents when receiving the signal from a SubSmell application. A SubSmell application is designed to read a SubSmell in the movie and decide to send signals to an olfactory display. A SubSmell application consists of four major components: 1) Movie Controlling, 2) SubSmell Reading, 3) Scent Releasing and 4) Olfactory Display Monitoring. We use Microsoft Visual Basic 6.0 to develop the user interface and the SubSmell components.

The experiment was done in order to assess the following qualities: 1) Usability: to prove that the system can read a SubSmell in the movie and release scent. 2) Efficiency: to show that the system can work with high accuracy.
\end{abstract}

\section{Introduction}

Nowadays, we can enjoy movies only by watching motion-pictures and listening to the sound which accompanies the movies. We use only two senses out of the five senses; which are sight and hearing. Movie makers try to improve the audience's feelings more by several ways. Regarding sight, they have studied optical nerves and how human eyes can receive color and pictures and how humans could feel distance of the objects. They have invented and developed many new technologies to capture and keep the best quality of images to make everything in the movies look more real so that the audience can feel it. One of those developed technologies was three-dimension imagining (3-D). The same way in hearing, they also studied the physical auditory nerve and also researched the way of how to keep the best sound quality for playing back to the audience.

T. Wada, F. Huang, and S. Lin (Eds.): PSIVT 2009, LNCS 5414, pp. 462 472, 2009.

(C) Springer-Verlag Berlin Heidelberg 2009 
One good example of 3-D technology was digital surround sound cinema. Movie makers can use only two senses of human beings, sight and hearing, which is a huge limitation for movies makers. They can not reach their goal to make movies as real as their imaginations. The solution of this limitation is to break the limitation and try to add more senses to the movies. To add more senses, the movies makers expect that they can make audiences have more imagination and feeling than they have ever had. They also introduce new senses of humans in their movies. Movies makers try to find the most important sense, which is easily added to multimedia among scent, taste and touch. Finally, they choose to add scent to the movies.

\section{Background}

Many researchers used many techniques to develop an olfactory display, such as Sensorama, head-mounted olfactory display (HMD), arm-worm olfactory display, DigiScents, Aromajet, ScentAir, Trisenx, Scent Collar etc. 1],2]. E.C. Tan et al. used an electromechanical system to control smell bottle to emit scent [3]. Takamichi et al. used 32 odor components flowed from bottles through a solenoid valve to the outlet [4, 5. Dong Wook Kim et al. used Aroma-Chip to build an olfactory display $[\underline{6}$.

From 1916 until now, there were many researchers trying to add scent to the movies and other multimedia with different methods such as;Scentovision, iSmell, Smellevision, Aroma-Rama, Scenoroma, Scentware, Odoram, Smell-OVision system and Scratch and Sniff cards. Each method had its limitations. This was very regrettable for the film owners and the audiences. Movies makers missed keeping their scent accompanying their movies and making their movies better. This also made movies audiences miss the scents. There were many movies which had scent as their main character such as perfume movies, cooking movies, etc. This was not only with the movies in the offline world, but also in the online world. Online movies also tried to accrue smell via the server and sniff smell to the website. Although some methods were not suitable for the movies, they were good for using with some advertisements. For instances scratch and sniff cards idea was used for business card. Several methods are still used in the real world and also they are trying to improve them and overcome their limitations. Smell-O-Vision is the most popular one, which is used to be a master model to create a better methodology by using the pipeline system to release scent to the audience. We might usually be able to see it in Scenoroma, Scentware etc [7] 8, 9] 10, 11] 12] 13].

\section{System Design}

This part describes the process of analysis and design, which describes the SubSmell system architecture overview, the system state transition diagram and system structure chart. The details of each element are described below. 


\subsection{SubSmell System Architecture Overview}

SubSmell is like a subtitle in the movie which the movie maker accompanies the text title to the movie, to describe what the actor/actress is saying. In this case we accompany scent to the movie to describe what scent is in that scene. We call it the SubSmell system. The SubSmell system works similarly to other media player application forms. We have to open the SubSmell application on our computer which is connected with an olfactory display. The system is divided into two parts: software and hardware. The software, SubSmell, is the main module which would read SubSmell in the movie, and then send the signal through ports of an olfactory display to release scent to the audiences. The olfactory display is a prototype that releases scent using fans. The audiences have only to play the movie which has the SubSmell logo with the SubSmell program, and then they would sniff the movie. The system overview is shown in Fig. 1]

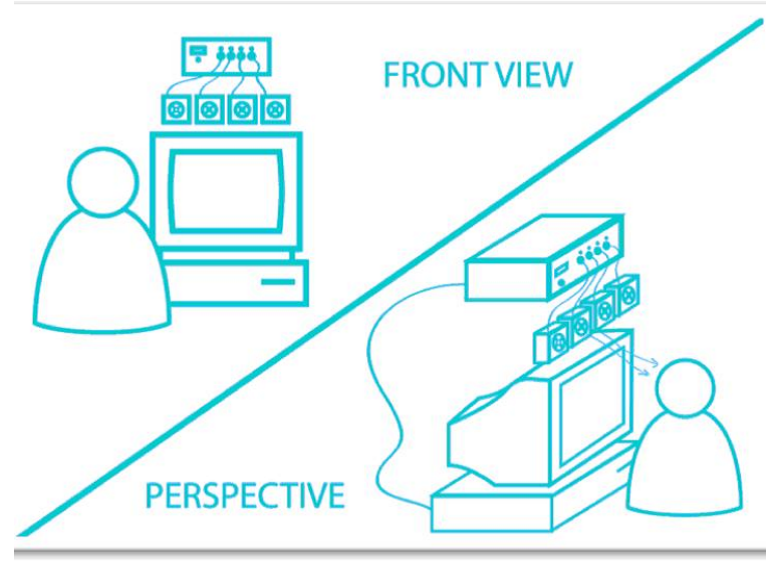

Fig. 1. SubSmell System Architecture Overview

SubSmell is a color SubSmell logo, as shown in Fig. 2(a), where each color would represent each scent. There are three smells for the SubSmell prototype system. We have three colors, which are red, green, and blue for representing these three smells. We also provide white color to represent clear scent and black color to represent the idle state. We use only one pixel in the SubSmell area, as shown in Fig. 2(b). The position of pixel that we get is very important. The pixel should be located in the middle of logo to get the clearest color of the pixel.

\subsection{System State Transition Diagram}

Based on Fig. 3, there are eight stages in the SubSmell System.

- State 1 "Start" when users open the SubSmell Application, I/O is called to be ready. Then go to stage 2 . 


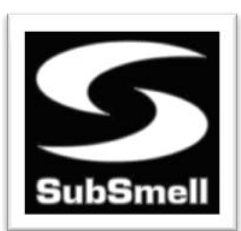

(a)

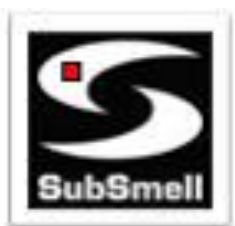

(b)

Fig. 2. (a) SubSmell Logo, (b) SubSmell Logo with suitable point to get a pixel

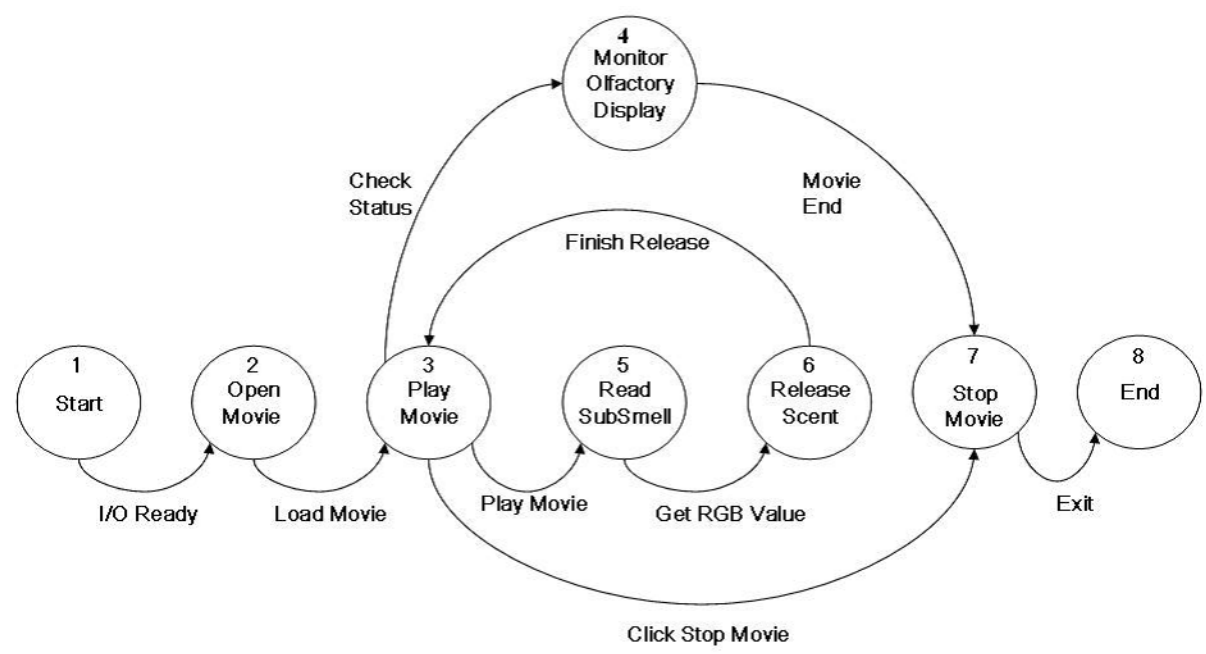

Fig. 3. SubSmell State Transition Diagram

- State 2 "Open Movie" when users choose a movie, the system will load it to the buffer and then go to stage 3 .

- State 3 "Play Movie" when users click on the play button, the movie is played and then we do stage 4 and stage 5 .

- State 4 "Monitor Olfactory Display" while the movie play timer is started and automatically checked, it shows the port status every millisecond. It goes to stage 7 ; the timer stops when the movie stops.

- State 5 "Read SubSmell" while the movie plays SubSmell, the system gets a pixel and converts to a RGB value, then go to stage 6 .

- State 6 "Release Scent" when it gets the RGB value, this stage checks the color condition and sends a signal to the odor machine. When it finishes releasing a scent, then go back to stage 3 .

- Stage 7 "Stop Movie" The users click on the stop button or the movie ends. Then stop and go to stage 8 .

- Stage 8 "End" when users exit the program, the system is shut down. 


\subsection{SubSmell System Structure Chart}

To provide a better understanding and more detail of each operation of the SubSmell system, we introduce the system structure chart (as shown in Fig. (4) and elaborate on how each model works. The SubSmell system consists of four main process modules. They are 1) Controlling Movies, 2) Read SubSmell, 3) Release Scent, and 4) Olfactory Display Monitoring. The second level of the structure chart gives the main tasks which have to be done in each component.

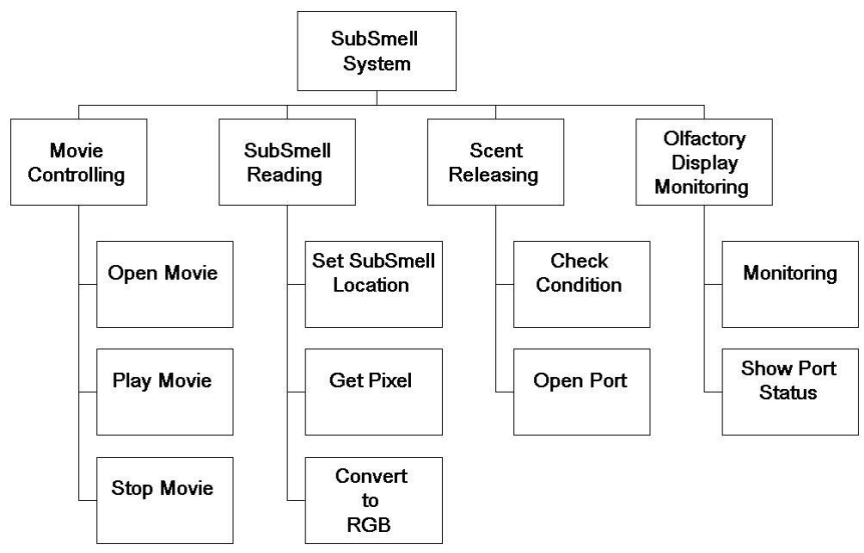

Fig. 4. SubSmell System Structure Chart

Movie Controlling. To use this application is similar to other media player applications. We are able to start the movie which we would like to see on the application program. We can play every movie which is encoded with Microsoft AVI. We have to take them carefully for encoding because it is the most sensitive part of the program. For example, a video file .AVI type can be encoded with Microsoft DV AVI or Microsoft AVI. We can only see the picture movie, which is encoded by Microsoft AVI. For other kinds of encoding, we cannot see the SubSmell system. Once we get Microsoft AVI movie, the next thing we have to consider is whether the movie has SubSmell or not? We can play the movie which has no SubSmell, but we would not smell the scent of the movie. However, if the movie has SubSmell and it is placed in the right location, the system can read it and understand what scent the movie should release in the frame, enabling us to sniff the movie.

For the SubSmell system, we provide three main control instructions for seeing the movie. They are in the form of buttons (as shown in Fig. (5) . The first is Open Movie, which is normally open. A pop-up window will appear for you to choose a movie file that you want. After choosing the file, the system will load the movie to the buffer and show the chosen file name in the title text field. The second control is Play Movie, which plays the chosen movie. The last control is Stop movie. You can click on this button to stop playing the movie. This stop is not 


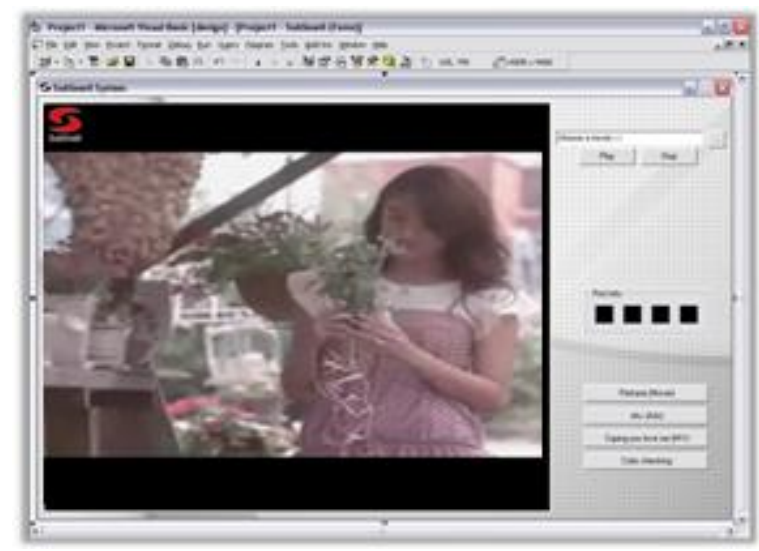

Fig. 5. SubSmell System User Interface

like other stops in most programs. It does not go to the end of the movie and then stop it. To stop is like to pause the movie and then play back or play other movie files.

SubSmell Reading. SubSmell logo is a big ' $\mathrm{S}$ ' character that comes from the first character of the project name, SubSmell. It is used as a sign to know what scent torelease at that time. We can attach scent in each scene that we want by putting a SubSmell logo in the top-left of the movie, as shown in Fig. 5. Each color of SubSmell represents each different scent.

At the start of the movie clip, it has to declare each color of SubSmell logo and what scent to release. For example, in the title of the movie it says red color, which represents Flower's scent, as shown in Fig. 6, allowing the user to know that he/she should put flower's scent in the red box of olfactory display. Then, when we play the movie and the system finds the red logo and sends the signal to the machine to open port number 1 (red box) we will smell the flower's scent.

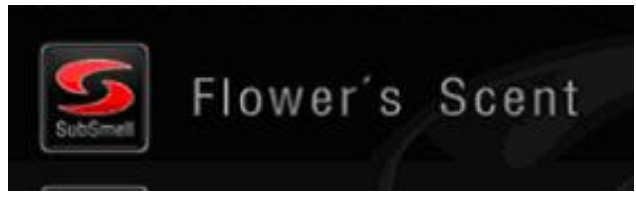

Fig. 6. SubSmell Logo big 'S'

In Fig. 17, we show five colors in the SubSmell logo for this project. They are Red, Green, Blue, White and Black. The color Red, Green, and Blue represent three different scents. The color White is for clearing scent that is released before. The color Black is for an idle state. 


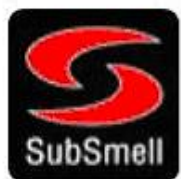

(Red)

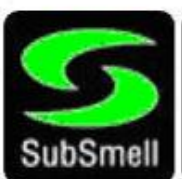

(Green)

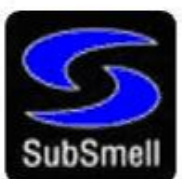

(Blue)

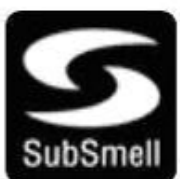

(White)

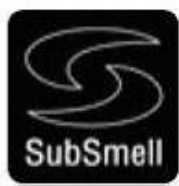

(Black)

Fig. 7. Five SubSmell logo with their colors

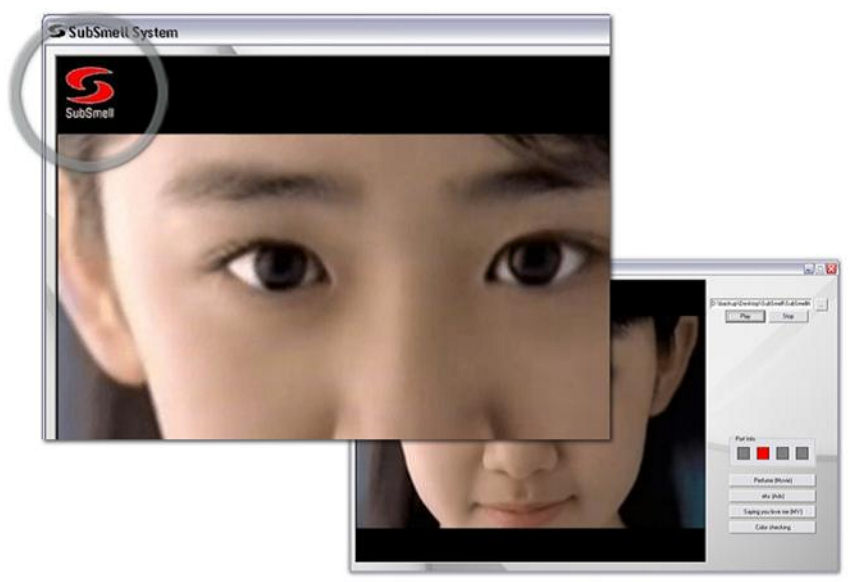

Fig. 8. Suitable location on SubSmell logo

The SubSmell reading does not use all areas of the SubSmell logo to identify the scent. It uses only one single pixel in a suitable location in SubSmell logo. The most suitable point on the SubSmell logo can be in the middle of the upper $\mathrm{S}$ or in the middle of the lower $\mathrm{S}$, as shown in Fig. 8. This process is called to get pixel. Then, we go to the next step to convert to RGB color mode because we have red, green, and blue for three different scents. It is very easy to check conditions about what scent we should release in the next step.

Scent Releasing. To release the scent of the movie, we should have a machine, as shown in Fig. 9, which can release each scent as we like. We have the example of the olfactory display with three scents and one clear state. The architecture of the olfactory display comes from a basic idea to keep the scent source in a small box, and then use a fan to blow out a scent, as shown in Fig. 10(a), and blow in to clear a scent, as shown in Fig. 10(b). For each scent, it has its own box and a fan which controls signaling via each port. We can thoroughly see how to invent this machine in an appendix part. For each scent, we are using liquid to make scent smell as real as perfume and cologne. Next, we drop liquid on the cotton and put the cotton inside the box. 


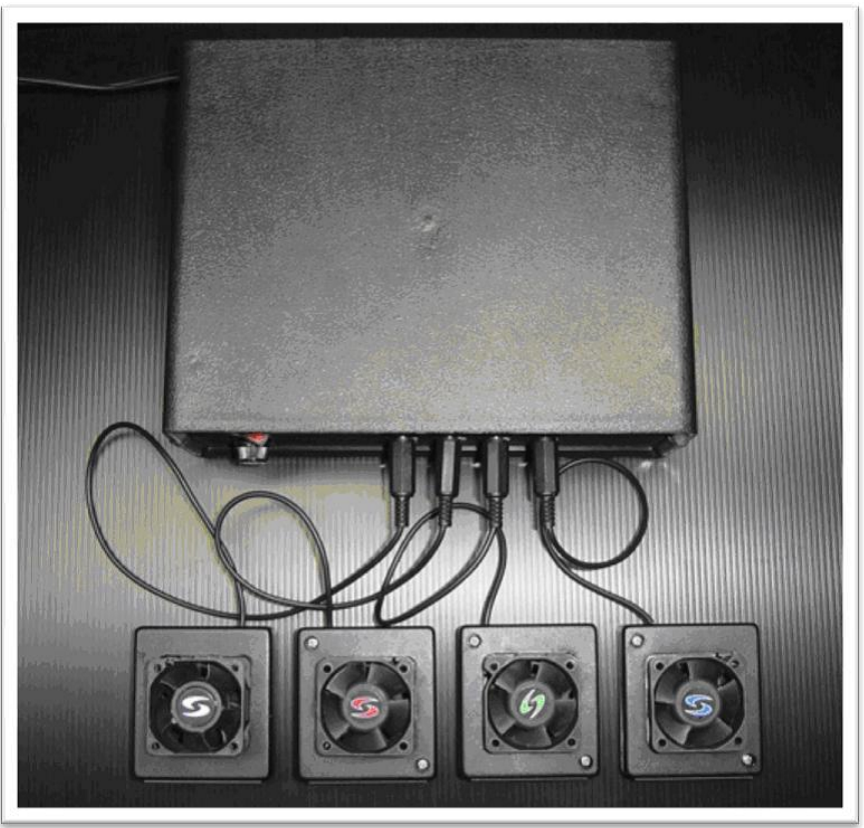

Fig. 9. Olfactory Display Model

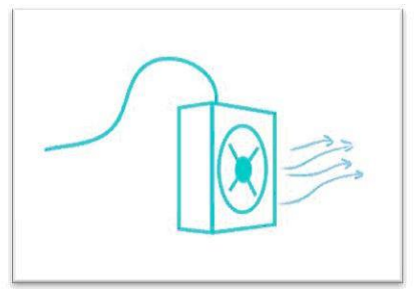

(a)

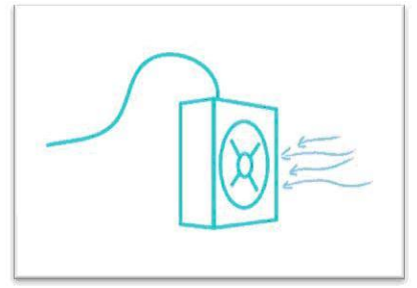

(b)

Fig. 10. Blow out fan (a) and Blow in fan (b)

As it is known that the user should put scent according to the declaration in the box that is provided, and then open the movie. From the last processes in the part of the SubSmell system application, we get an RGB color pixel for each time frame from the last process. We bring this pixel to check the condition that belongs to which color, red, green, or blue. We cluster by checking Red, Green, or Blue has the most in this pixel, so we consider which port we should send signal for opening the fan and blowing out a scent, as shown in Fig. 11. 


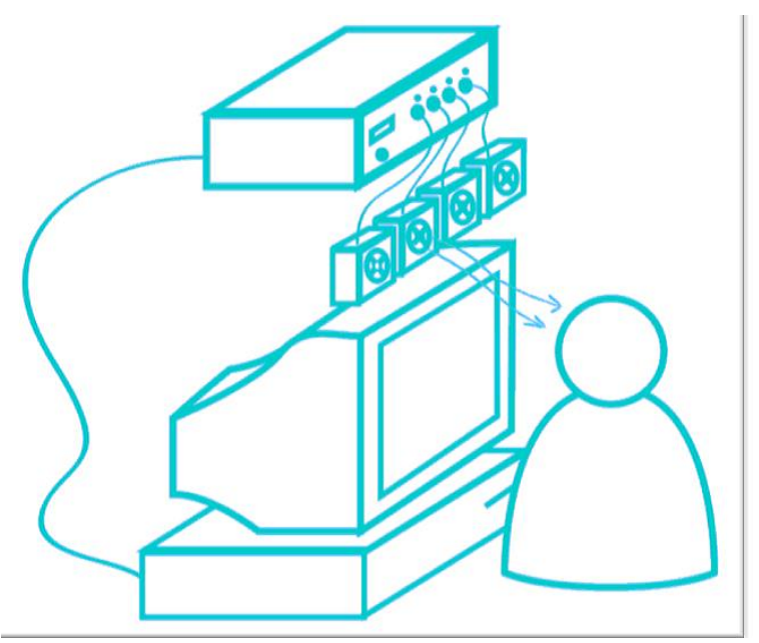

Fig. 11. Flow of Scent to the User

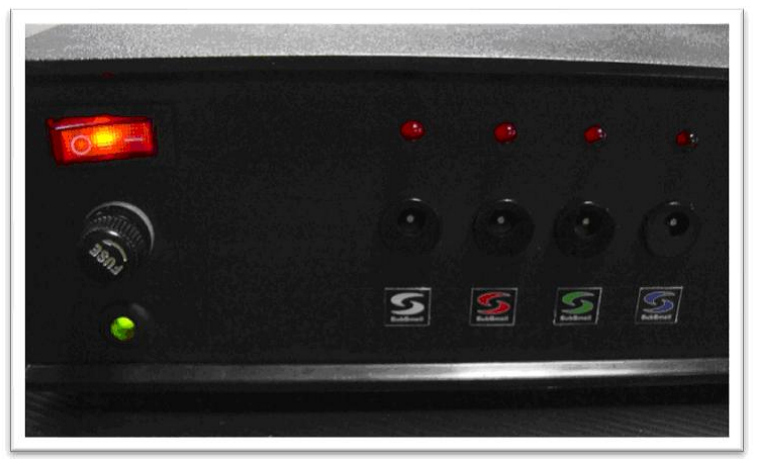

Fig. 12. The LED light to show a status of the olfactory display

Olfactory Display Monitoring. To monitor the status of the olfactory display is an important process to do, because we would be able to see the flow of scent along the scene and check the performance of how fast and accurate the system detects the SubSmell system. We check every second which port is opening. Then, we are able to compare with our eye which is accurate or not. In case we have seen the Green SubSmell appearing at the top-left of the movie, but the Red port is opening. This means the system is wrong because it makes a mistake in classifying the detected color pixel.

Figure 12 shows the status of the olfactory display by using the LED light. The method to verify the status of the olfactory display is to check every second the opening port, which shows the light on the olfactory machine. 


\section{Testing and Evaluation}

In this part, we test the system with various conditions in various cases to measure its efficiency and effectiveness. After that we evaluate the system so that it can be used in the real world.

This part presents experimentation on the SubSmell system, which is developed and based on the concepts and the design mentioned in the previous section. In this system, the experiments were focused on its usability and effectiveness.

Usability testing was to prove whether the system was capable of performing the proposed functions as we had mentioned earlier. The effectiveness test determined the correctness of the system and whether the system results could be used in real life.

\subsection{Usability}

The first thing was to use the SubSmell system to open and play the movie. We tested this function by selecting many kinds of movies that were encoded by .AVI code. We had known that the limitation of the SubSmell system was its ability to open and play only .AVI files. When users used Adobe Premiere or any other video editing program to make SubSmell Movie, they should export by using only "Microsoft AVI" to open the file. All of the data tested were selected and played until the end. The testing results are shown in Table 1.

Table 1. Testing Data Details

\begin{tabular}{|c|c|c|c|c|}
\hline Title & Type & Length & Use $\mathrm{n}$ scent(s) & Play result \\
\hline Perfume & Movie & 13 mins 31 secs & 5 & Great \\
\hline Saying you love me & Music video & 4 mins 2 secs & 5 & Great \\
\hline Eu: cologne & Advertisement & 33 secs & 5 & Great \\
\hline
\end{tabular}

The second thing we had checked was the readability of the SubSmell system and how well it could read each pixel in each frame, and what value of each color it got. We had tested the system to read each SubSmell color in the specified position and corrected the number that the System read.

\subsection{Effectiveness}

To measure how well the SubSmell system could release scent, we had tested the system by using the SubSmell movie and observed at the olfactory display port. The olfactory display had four fans, out of three fans were used to blow out the scent and the other one to blow into the clear scent. Table 2 shows all SubSmell colors with control port number and event of a fan. 
Table 2. Color and Port Information

\begin{tabular}{|c|l|c|}
\hline Color & Port number & Event \\
\hline Red & Port1 $(\& \mathrm{H} 8)$ & Blow out \\
\hline Green & Port2 $(\& \mathrm{H} 4)$ & Blow out \\
\hline Blue & Port3 $(\& \mathrm{H} 2)$ & Blow out \\
\hline White & Port4 $(\& \mathrm{H} 1)$ & Blow in \\
\hline Black & Port0 $(\& \mathrm{H} 0)$ & Idle \\
\hline
\end{tabular}

\section{Conclusion}

The SubSmell system has been proved to be usable and effective as we have described in the testing and evaluation section. We may conclude that the SubSmell system supports the following. 1) the movies audiences can view the pictures with aesthetic quality, which means they can see the scenes and smell the scents simultaneously, and 2) the audiences get more detail of the movie, either scene, sound or smell, which makes it more entertaining and interactive.

\section{References}

[1] Chen, Y.: Olfactory display: development and application in virtual reality therapy. In: Pan, Z., Cheok, D.A.D., Haller, M., Lau, R., Saito, H., Liang, R. (eds.) ICAT 2006. LNCS, vol. 4282. Springer, Heidelberg (2006)

[2] Washburn, D.A., Jones, L.M.: Could olfactory displays improve data visualization? Computer in Science \& Engineering (November/December 2004)

[3] Tan, E.C., Wahab, A., Goh, G.H., Wong, S.H.: PC-Controlled Scent System. IEEE transactions on Consumer Electronics (December 1998)

[4] Nakamoto, T., Otaguro, S., Kinoshita, M., Nakamaha, M., Ohinishi, K., Ishida, T.: Cooking up an Interactive Olfactory Game Display. IEEE Transactions on Computer Graphics and Applications (January/February 2008)

[5] Project Scent Projector - ATR MIS (accessed date: April 25, 2008), http://www.mis.atr.jp/past/sem/scent.html

[6] Kim, D.W., Nishimoto, K., Kunifuji, S.: An Editing and Displaying System of Olfactory Information for the Home Video. Springer, Heidelberg (2006)

[7] Smell-O-Vision (creation date: unknown, last modified date: unknown accessed date: January 20, 2007), http://en.wikipedia.org/wiki/Smell-o-vision

[8] Odorama (creation date: unknown, last modified date: unknown, accessed date: January 20, 2007), http://en.wikipedia.org/wiki/Odorama

[9] iSmell (creation date: unknown, last modified date: unknown, accessed date: January 20, 2007), http://en.wikipedia.org/wiki/ISmell

[10] Sensoroma (creation date: unknown, last modified date: unknown, accessed date: January 20, 2007), http://www.sensomatic.com/sensorama/.

[11] Kaori Web (creation date: unknown, last modified date: unknown, accessed date: January 20, 2007), http://gizmodo.com/archives/kaori-web-internetsmellovision-018646.php

[12] Digital Scentware? (creation date: unknown, last modified date: unknown, accessed date: January 20, 2007),

http://findarticles.com/p/articles/mi_pwwi/is_200005/-ai_mark15009826

[13] Scentware (creation date: unknown, last modified date: unknown, accessed date: January 20, 2007), http://www.ediblecomputer.com/040802.html 